\title{
ABCG1 Gene
}

National Cancer Institute

\section{Source}

National Cancer Institute. ABCG1 Gene. NCI Thesaurus. Code C113598.

This gene is involved in lipid transport. 